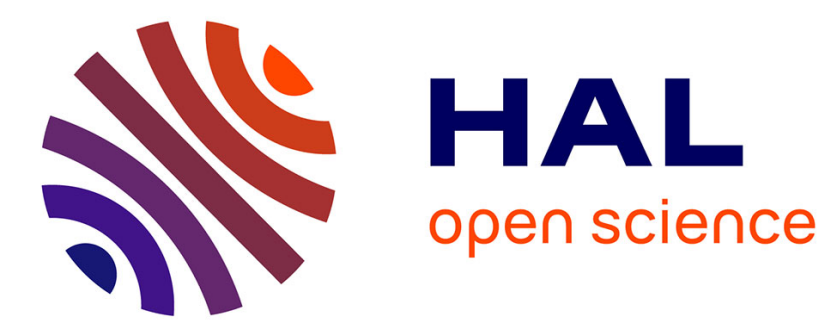

\title{
HF-VHF Electromagnetic Emissions from Collisions of Sprite Streamers
}

\author{
M. B Garnung, Sébastien Celestin, T. Farges
}

\section{To cite this version:}

M. B Garnung, Sébastien Celestin, T. Farges. HF-VHF Electromagnetic Emissions from Collisions of Sprite Streamers. Journal of Geophysical Research Space Physics, 2021, 126 (6), pp.e2020JA028824. 10.1029/2020JA028824 . insu-03256010

\section{HAL Id: insu-03256010 https://hal-insu.archives-ouvertes.fr/insu-03256010}

Submitted on 10 Jun 2021

HAL is a multi-disciplinary open access archive for the deposit and dissemination of scientific research documents, whether they are published or not. The documents may come from teaching and research institutions in France or abroad, or from public or private research centers.
L'archive ouverte pluridisciplinaire $\mathbf{H A L}$, est destinée au dépôt et à la diffusion de documents scientifiques de niveau recherche, publiés ou non, émanant des établissements d'enseignement et de recherche français ou étrangers, des laboratoires publics ou privés. 


\title{
HF-VHF Electromagnetic Emissions from Collisions of Sprite Streamers
}

\author{
M. B. Garnung ${ }^{1,2}$, S. Celestin ${ }^{1}$, and T. Farges ${ }^{2}$ \\ ${ }^{1}$ LPC2E, University of Orleans, CNRS, Orleans, France \\ ${ }^{2}$ CEA, DAM, DIF, F-91297, Arpajon, France
}

\section{Key Points:}

- EM emissions produced by a collision between two sprite streamers with opposite polarities are predicted using streamer and antenna models.

- Calculated radiated power from these EM emissions is compared to DEMETER, TARANIS, and FORTE radio instruments sensitivities.

- Sensitive ground-based instruments such as radiotelescopes (e.g., NenuFAR) may detect these emissions.

Corresponding author: M. B. Garnung, matthieu.garnung@cnrs-orleans.fr

This article has been accepted for publication and ${ }^{-1}$ undergone full peer review but has not been through the copyediting, typesetting, pagination and proofreading process, which may lead to differences between this version and the Version of Record. Please cite this article as doi: 10.1029/2020JA028824.

This article is protected by copyright. All rights reserved. 


\begin{abstract}
Sprites are complex transient plasma discharges that consist of many plasma filaments named streamers. They are produced high above thunderstorms. Sprites are known to produce electromagnetic radiation observed typically in the extremely low (ELF), ultra low (ULF), to as high as medium frequency (MF) radio bands. Recent research work showed that head-on streamer collisions lead to a reinforcement of the electric field over a short time scale, typically a few picoseconds at ground-level. The use of the similarity laws leads to a corresponding time scale on the order of a fraction of a microsecond at $50 \mathrm{~km}$ altitude, which opens the eventuality for HF-VHF emissions from sprites. In this paper, using a multifluid streamer model paired with an antenna model assimilating the streamer as a straight segment. We simulate head-on collision between two streamers with opposite polarities in order to evaluate their electromagnetic emissions. We report numerical prediction of the electromagnetic signature for 50,60,70, and $80 \mathrm{~km}$ altitudes. The magnetic field radiated varies over 4 orders of magnitude, between less than $0.1 \mathrm{fT}$ to $7 \mathrm{pT}$. Comparing the spectral density from the head-on collision between two streamers with IME-HF (TARANIS), ICE (DEMETER), and FORTE RF payload, we find that IME-HF and ICE could detect these signatures. We compare these results with sensitive ground-based instruments like the radiotelescope NenuFAR, and show that detections of such events might be possible with this type of fast and sensitive radiotelescopes.
\end{abstract}

\title{
1 Introduction
}

Sprites are sudden and bright luminous events occuring above thunderclouds between $40 \mathrm{~km}$ and $90 \mathrm{~km}$ altitude. They have been observed for the first time in 1989 (Franz et al., 1990) during the test of a TV camera in low light condition. Sprites are composed of many plasma filaments named streamers, which radiate electromagnetic emissions. Cummer et al. (1998) observed electromagnetic emission in the extremely low frequency (ELF) to ultra low frequency (ULF) range due to electric currents flowing in the body of sprites. They evaluated that the electromagnetic energy in the ELF range is comparable to that of the parent cloud-to-ground (CG) lightning discharge. Füllekrug et al. (2001) confirmed these observations with radio and optical records of sprites with long-time delays relative to their parent lightning discharges. Füllekrug et al. (2010) recorded emissions in the low frequency (LF) range, which are temporally coincident with the sprite light emission. Farges and Blanc (2011) reported electromagnetic radiation in the medium frequency 
(MF) range during sprites events. Qin et al. (2012) demonstrated the importance of the local air density on the emission frequency for a single-headed streamer and proposed that LF emissions are associated with streamer expansion processes.

In a different context, Ihaddadene and Celestin (2015) showed that collisions between streamer discharges at air at ground-level with opposite polarities would lead to strong electric field variations over a duration on the order of a dozen of picoseconds under high electric fields. This was later confirmed through the use of different models (e.g., Köhn et al., 2017; Babich \& Bochkov, 2017; Luque, 2017; Shi et al., 2019). Luque (2017) and Shi et al. (2019) reported that such streamer collisions at ground-level should produce electromagnetic emission in the ultra high frequency (UHF i.e., $300 \mathrm{MHz}-3 \mathrm{GHz}$ ) range. Using similarity laws (e.g., Pasko et al., 1998; Pasko, 2006; Qin \& Pasko, 2015) to scale this typical duration of $\sim 10 \mathrm{ps}$ at an altitude of $60 \mathrm{~km}$, one finds a typical time scale on the order of a few tens of nanoseconds suggesting that electromagnetic emission in the high frequency (HF) and very high frequency (VHF) bands (respectively $3 \mathrm{MHz}-$ $30 \mathrm{MHz}$ and $30 \mathrm{MHz}-300 \mathrm{MHz}$ ) might be produced. These emissions would be observable from space for frequencies above the ionospheric cutoff. It is worth mentioning that collisions between streamers seem common in sprites as it can be seen on pictures reported in (e.g., Gerken et al., 2000; Cummer et al., 2006). Indeed, one can also observe a change in the filament shape and brightness in the vicinity of intersections, indicative of actual collisions. We cite an observation, which is striking regarding the importance and frequency of streamer collisions in sprites: this result was first presented at the AGU Fall Meeting 2019 in San Francisco (McHarg et al., 2019).

The original idea of using streamers with opposite polarities came from the scaling at timescales found by (Ihaddadene \& Celestin, 2015) to sprite altitudes. It is clear from high-speed video observations that a great number of collisions between streamers of opposite polarities do occur, despite the fact that the occurrence frequency is not quantified yet (e.g., McHarg et al., 2019). Other kinds of collisional geometries leading to rapid changes in the electric current can be envisioned, such as the collision of streamers with inhomogeneities (Luque \& Gordillo-Vázquez, 2011; Ihaddadene et al., 2019) or the connection of a streamer with the oppositely charged tail of another streamer (Luque \& Ebert, 2010). 
TARANIS (Lefeuvre et al., 2008) was a satellite mission funded by the French space agency (CNES) dedicated to the study of impulsive transfers of energy between the troposphere and the near-Earth environment, which are manifested by Transient Luminous Events (TLEs) and Terrestrial Gamma rays Flashes (TGFs) produced by thunderstorm activity. TARANIS had several types of sensors to observe TLEs and TGFs. In this paper, we are particularly interested in the electric field instrument named Instrument de Mesure du champ Electrique (IME), and the magnetic field instrument named Instrument de Mesure du champ Magnétique (IMM). IME includes two antennas, IME-BF for the low frequencies from DC up to $1 \mathrm{MHz}$ and IME-HF for the high frequencies from $100 \mathrm{kHz}$ up to $35 \mathrm{MHz}$. IMM is a tri-axis magnetometer, so-called search-coil, designed to measure the magnetic field variation between $5 \mathrm{~Hz}$ and $1 \mathrm{MHz}$. In addition, the instrument MCP (MicroCameras and Photometers) is made up of 2 cameras and 4 photometers designed to detect and characterize TLEs (Farges et al., 2018).

Unfortunately, TARANIS has never reached its orbit due to launch failure on November 17th, 2020. We hence to compare with previous radio instruments from other missions dedicated to lightning observations in order to evaluate the possibility to use previous work and possibly future work to conduct such studies. We then use the radio instrument ICE from the DEMETER mission (Berthelier et al., 2006) and the FORTE's radio payload (Jacobson et al., 1999). These two missions have the advantage to have a similar orbit trajectory to that TARANIS would have had.

The DEMETER mission was to provide a nearly continuous survey of natural electrostatic, electromagnetic emissions, and ionospheric irregularities that can be related to seismic activity (Parrot et al., 2006). In this paper, we focus on the radio instrument ICE (Berthelier et al., 2006), which relies on four spherical sensors mounted at the ends of four antenna arms. It operates over a wide frequency range from DC to $3.175 \mathrm{MHz}$. Note that the DEMETER mission had also a magnetic instrument named IMSC that we do not consider in the present study because of a weak sensitivity.

The FORTE mission was dedicated to the study of lightning from space with optical and radio payloads (Jacobson et al., 1999). As for the DEMETER mission, we focus on the RF payload that is composed of two tunable receivers in the frequency range 20-300 MHz. 
The purpose of this work is to characterize the electromagnetic radiation expected to be produced by collisions of streamers with opposite polarities, and to study its detectability by TARANIS, DEMETER, FORTE, and ground-based instruments such as the radiotelescope NenuFAR (Zarka et al., 2012, 2015).

\section{Methods}

In this work, we use a streamer model based on a 3-D axisymmetric fluid approach (e.g., Liu \& Pasko, 2004). It consists in solving Poisson's equation (1) coupled with driftdiffusion equations (2)-(4). Poisson's equation is written as:

$$
\nabla^{2} \phi=-\frac{q_{e}}{\epsilon_{0}}\left(n_{p}-n_{e}-n_{n}\right)
$$

where $\phi$ is the electric potential, $q_{e}$ is the elementary charge, $\epsilon_{0}$ is the vacuum permittivity, and $n_{p}, n_{e}$, and $n_{n}$ are the positive ion, electron, and negative ion densities, respectively. Equation (1) is numerically solved using a Successive Over-Relaxation (SOR) method (Press et al., 2007) coupled with Chebyshev's acceleration method (Golub \& Van Loan, 1996). Drift-diffusion equations (2), (3), and (4) describe the fluid motion and the local evolution of the electron, positive ion, and negative ion populations, respectively:

$$
\begin{gathered}
\frac{\partial n_{e}}{\partial t}+\vec{\nabla} \cdot\left(n_{e} \overrightarrow{v_{e}}-D_{e} \vec{\nabla} n_{e}\right)=\left(\nu_{i}-\nu_{a}\right) n_{e}+S_{p h} \\
\frac{\partial n_{p}}{\partial t}+\vec{\nabla} \cdot\left(n_{p} \overrightarrow{v_{p}}-D_{p} \vec{\nabla} n_{p}\right)=\nu_{i} n_{e}+S_{p h} \\
\frac{\partial n_{n}}{\partial t}=\nu_{a} n_{e}
\end{gathered}
$$

where $n_{k}$ is the density, $v_{k}$ is the velocity, $D_{k}$ is the diffusion coefficient, and $k$ is $e$ for electrons, $p$ for positive ions, and $n$ for negative ions. $\nu_{i}$ is the ionization frequency while $\nu_{a}$ is the the sum of the two- and three-body electron attachment frequencies. These frequencies are calculated from Morrow and Lowke (1997). $S_{p h}$ is the rate of electron-ion pair production due to photoionization processes evaluated using the three-group $\mathrm{SP}_{3}$ model (Bourdon et al., 2007). Drift-diffusion equations are computed numerically through a basic upwind scheme (low-order) combined with an $8^{\text {th }}$ high-order scheme using the Flux Corrected Transport technique (Zalesak, 1979).

The numerical grid has a size of $151 \times 1681$ for a resolution $8 \frac{N}{N_{0}} \mu \mathrm{m}$, where $N$ is the local neutral density of air and $N_{0}$ is the air density at ground level. The simulation is conducted at an altitude of $70 \mathrm{~km}$ under a homogeneous electric field of $40 \frac{N}{N_{0}} \mathrm{kV} / \mathrm{cm}$. As the length of the simulated domain along the $z$-direction is small $(<200 \mathrm{~m})$, we ne- 
glect the variation of the air density as function of the altitude. Two gaussian neutral plasma seeds with a characteristic radius $\sigma=3 \mathrm{~m}$ are placed at $75.6 \mathrm{~m}$ and $122.8 \mathrm{~m}$ away from the right border so as to produce two double-headed streamers, such that at $99 \mathrm{~m}$ (i.e., in the middle of the simulation domain) a head-on collision occurs between a negative and a positive streamer as shown in Figure 1. The initial density distribution is

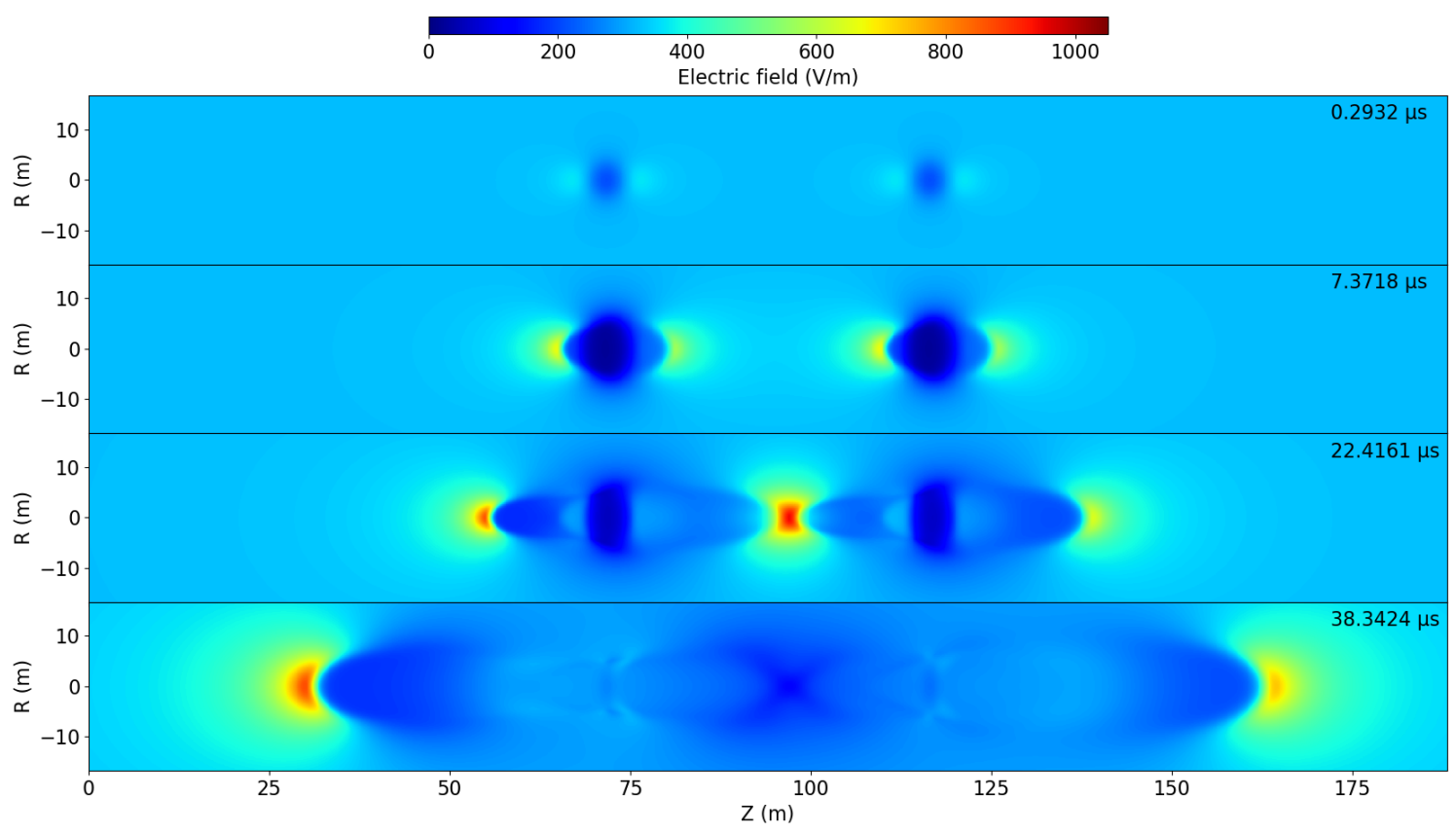

Figure 1. Four 2-D cross-sectional views of the absolute value of the axial component of the electric field for two double-headed streamers at an altitude of $70 \mathrm{~km}$ under a Laplacian electric field of $40 \frac{N}{N_{0}} \mathrm{kV} / \mathrm{cm}$. The time for each snapshot is given at the top-right corner of each panel while the electric field is encoded with the colorbar. The head-on collision between the two double-headed streamers (identified as the moment of time the electric field reaches its maximum) occurs at $22.12 \mu \mathrm{s}$.

137

written as:

$$
n_{i, j}=A\left[\exp \left(-\frac{r_{i}^{2}+\left(z_{j}-z_{0}\right)^{2}}{\sigma^{2}}\right)+\exp \left(-\frac{r_{i}^{2}+\left(z_{j}-z_{1}\right)^{2}}{\sigma^{2}}\right)\right]
$$

where $A$ is the peak electron density, $z_{0}$ and $z_{1}$ are the positions of the maximum density of the seeds. In this work, at $70 \mathrm{~km}$ altitude, $A=4.6 \times 10^{11} \mathrm{~m}^{-3}, z_{0}=75.6 \mathrm{~m}$, and $z_{1}=122.8 \mathrm{~m}$. 

observational geometry is sketched in Figure 2.

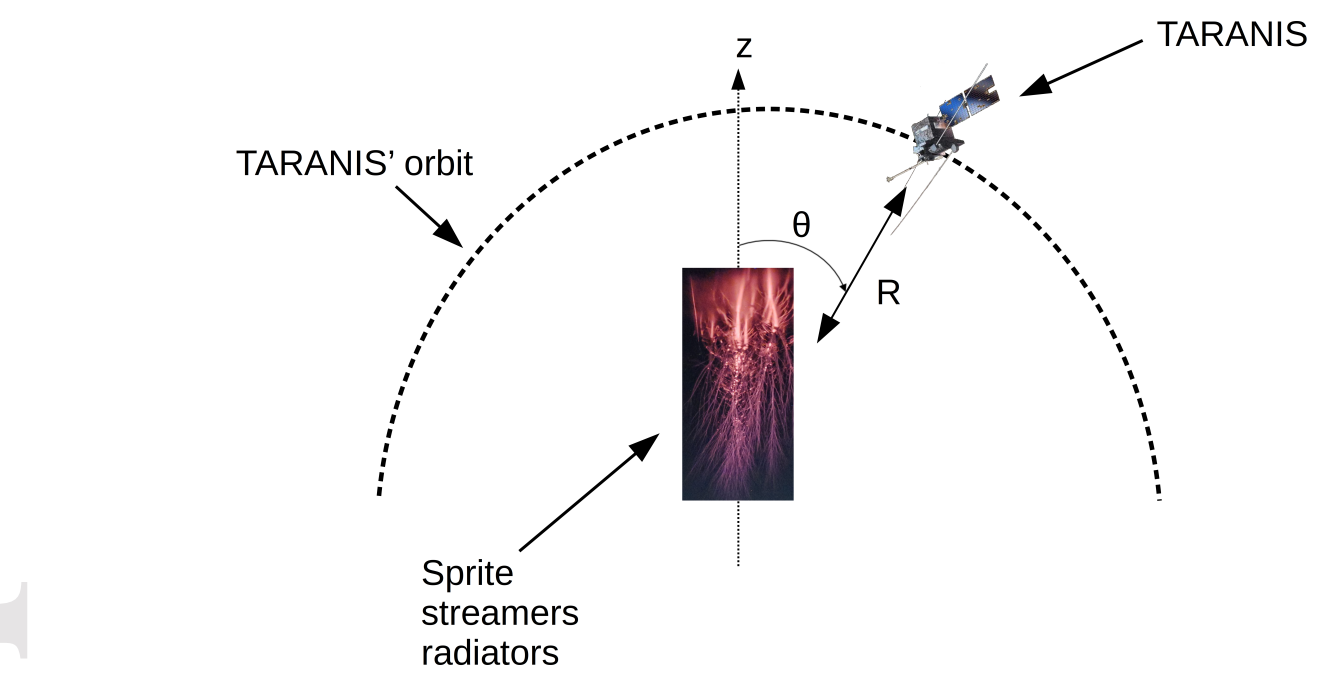

where $H_{1}$ and $H_{2}$ are the lower and upper edges of the simulation domain so that the length of the antenna is given by $H_{2}-H_{1}$. The variable $\theta$ is the polar angle between the observer (the spacecraft) and the streamers system (see Figure 2). As a sprite event occurs over a short timescale, we consider that $\theta$ is constant, and for the sake of simplicity and to maximize the produced magnetic field, we set it to $90^{\circ}$. The variable $R$ is the distance between the spacecraft and the streamers system. In this study, we consider the case of spacecraft orbiting at an altitude of $600 \mathrm{~km}$. The quantity $i(z, t)$ is the total current resulting from the summation of the fluid current produced by ions and electrons, and the displacement current created by the temporal variation of the electric field. The

Figure 2. Observational geometry used in our model. Streamers are considered as straight antennas. The spacecraft is at a distance $R$ from the streamers system, which is localized at a given altitude, and making an angle $\theta$ with respect to the spacecraft. Credits for the sprite picture: Stéphane Vetter (2019). ulation. We resample the obtained radiated magnetic field at the lowest timestep used 
during the numerical simulation in a regular fashion through a cubic interpolation. For a simulation at $70 \mathrm{~km}$ altitude, this lowest timestep is $2.5 \mathrm{~ns}$.

The electric field radiated by the antenna is evaluated using the approximation $E=$ $c B$ as the receiver is far away from the source. Indeed, a sprite streamer can be found between $40 \mathrm{~km}$ and $90 \mathrm{~km}$ altitude while typical satellites in low Earth orbit are at $\sim 600$ $\mathrm{km}$ altitude. Furthermore, we neglect the first term in equation (6) giving that the receiver is far away from the source. This assumption is verified if both terms in the equation (6) compare such that:

$$
\frac{1}{R} i\left(z, t-\frac{R}{c}\right) \ll \frac{1}{c} \frac{\partial i\left(z, t-\frac{R}{c}\right)}{\partial t}
$$

after straightforward manipulation, we obtain:

$$
R \gg c \frac{i\left(z, t-\frac{R}{c}\right)}{\frac{\partial i\left(z, t-\frac{R}{c}\right)}{\partial t}}
$$

and through a dimensional analysis, one obtains:

$$
R \gg c \Delta t
$$

The typical duration of the entire simulation, including propagation of double-headed streamers and their head-on collision at $70 \mathrm{~km}$ is around $20 \mu \mathrm{s}$. We hence get $c \Delta t \sim$ $6 \mathrm{~km}$, which is about 100 times lower than the streamer-receiver distance $(\sim 600 \mathrm{~km})$ considered in the study. The assumption neglecting the first term of the equation (6) is therefore deemed valid in the present study.

Gerken et al. (2000) show that streamers can have transverse extents of about 150 $\mathrm{m}$ above $60 \mathrm{~km}$ altitude. Such streamers are therefore much wider than those usually obtained in simulations and presumably would carry much stronger electric currents. Indeed, the radius of the streamers shown in Figure 1 is $\sim 5 \mathrm{~m}$. We hence consider a factor of $\sim 30$ between such simulated sprite streamers and those in reality above $60 \mathrm{~km}$. This difference in the streamer radius needs to be accompanied by an increase in the electric current proportional to the increase in the transverse area $\left(30^{2}=900\right)$. As part of a preliminary work, through numerical simulations we have verified that such scaling is physical and that the corresponding streamers do follow the dynamics of smaller ones. However, those simulations are extremely resources- and time-consuming if to be pursued with a high grid resolution. In this work, we therefore choose to use this simple factor of $30^{2}$ to obtain the current carried by high-altitude sprite streamers. Note that the 
difference in size between real sprite streamers and simulated ones is also confirmed by Liu et al. (2009) who found 4 orders of magnitudes between simulated and observed streamer brightnesses (brightness should be proportional to the streamer volume $\left(\sim 30^{3}\right)$ ) and McHarg et al. (2010) showed that a typical sprite streamer tip is $193 \mathrm{~m}$, but found much greater radii for splitting streamers.

\section{Results and Discussion}

Figure 1 shows a 2-D cross-sectional view of the electric field at the moment of the head-on collision between two double-headed streamers at $70 \mathrm{~km}$. The collision occurs at $z \sim 100 \mathrm{~m}$. The simulation domain is scaled at other altitudes using similarity laws. As part of preliminary work for the present study, we have verified the validity of this scaling-based method by realizing simulations at various altitudes. Figure 3 shows the

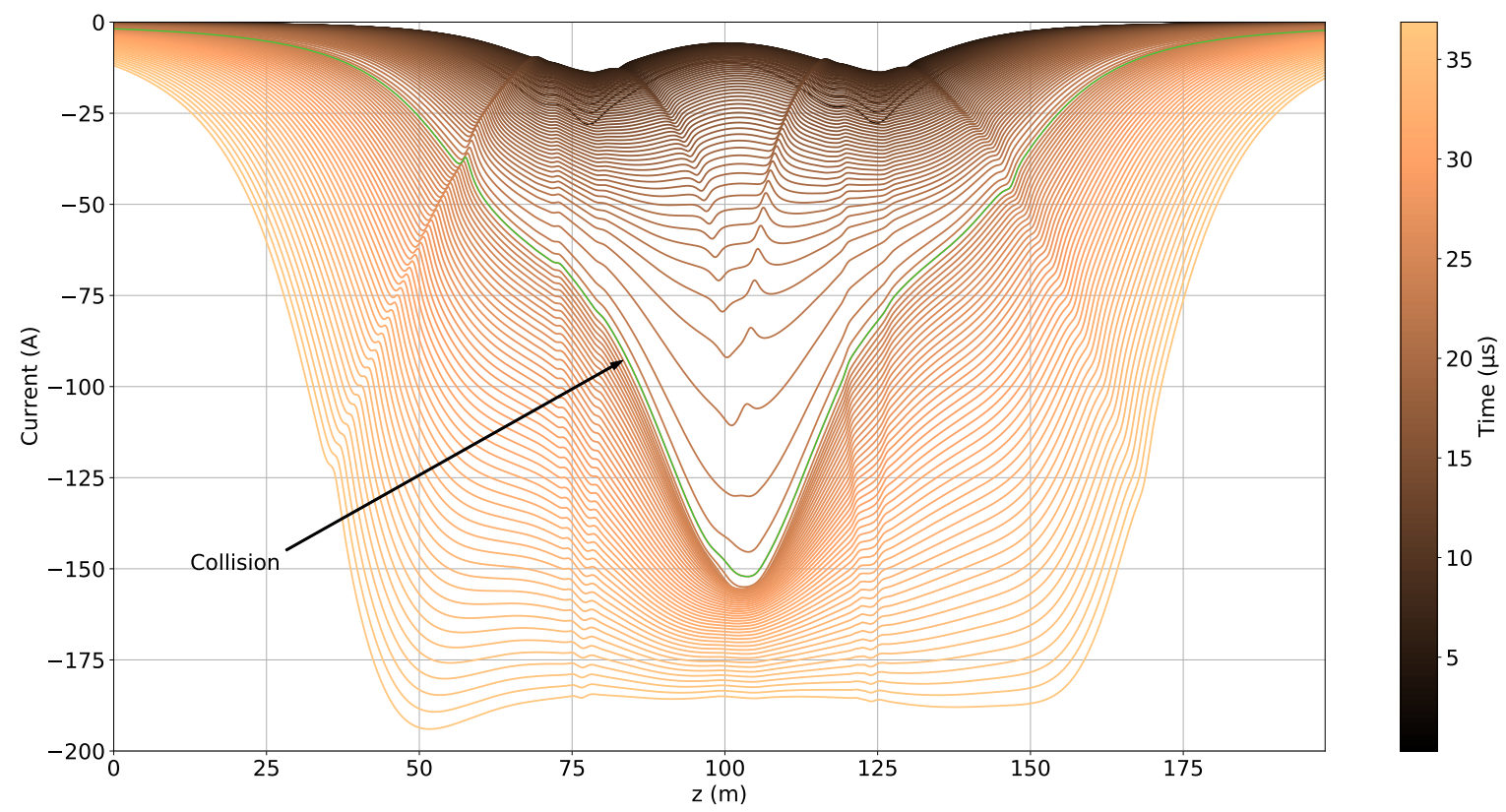

Figure 3. Total electric current along the axis of the domain as a function of position (lines are separated by a step of $0.29 \mu \mathrm{s}$ ) for the collision between two double-headed streamers illustrated in Figure 1. The time associated with the collision is defined as the time at which the electric field reaches its maximum.

temporal evolution of the total current $i(z, t)$ (Equation (6)) before, during, and after the collision at $70 \mathrm{~km}$ (Figure 1). It illustrates the increase of the current during the ex- 
pansion of the two double-headed streamers before the collision (above the green curve), the strong variation of the current during the collision (green curve), and the increase of the current after the collision (below the green curve).

Figure 4a shows the magnetic field waveform radiated by streamers for collisions occurring at different altitudes as it would be observed by satellite when ignoring the dispersion of the signal through the ionosphere. We can separate each colored curve in three parts. The first part is before the collision. During that stage, the increase of the magnetic field is due to an increase of the current in time resulting from the expansion of the two double-headed streamer, as first reported by Qin et al. (2012). The second stage corresponds to the interaction between streamers. During the interaction, the electric field reaches up to $271 \frac{N}{N_{0}} \mathrm{kV} / \mathrm{cm}$ leading to a strong increase in the electron density over a few nanoseconds, and then a strong variation of the current moment over a short time scale leading to a significant increase of the magnetic field illustrated by peaks in Figure $4 \mathrm{a}$. The increase of the electron density involves an increase of conductivity, which then leads to a sudden decrease of the electric field. Following the collision, we obtain a single double-headed streamer resulting from the merging of the two double-headed streamers. In the third stage, the total current is still increasing because of the expansion of the single double-headed streamer (Qin et al., 2012), as during the first stage. This expansion is due to the presence of a homogeneous electric field above the stability field for streamer propagation (e.g., Liu et al., 2009).

We conducted a simulation using the same setup with a single double-headed streamer in the aim to make a control experiment. Comparison with this no-collision case is shown in Figure 4a. In this figure the magnetic field in the control simulation (no-collision case) is multiplied by two to represent the magnetic field radiated by two non-interacting streamers. This comparison reveals that in the absence of a collision, the radiated magnetic field is around $1 \mathrm{pT}$ at the position of the satellite, while in the case with collision we found $7 \mathrm{pT}$ at $70 \mathrm{~km}$ altitude. The results for $50 \mathrm{~km}, 60 \mathrm{~km}$, and $80 \mathrm{~km}$ are scaled using similarity laws. The sprite-observer distance stays at $600 \mathrm{~km}$ for all cases.

In Figure 4b, we show the spectral density of electric fields radiated by two streamer systems with and without collisions scaled at different altitudes. For each spectral density shown in Figure 4b, we observe three spectral regions for the case with collisions (plain curves). The first region corresponds to the linearly decreasing part of the spectral den- 
sity and stops before the first significant decrease in the spectral density of the electric field in Figure 4b. The frequency range for this region starts from $\sim 630 \mathrm{kHz}$ up to $\sim 1.2$ $\mathrm{MHz}$ for an altitude of $50 \mathrm{~km}$. For an altitude of $60 \mathrm{~km}$, the frequency range spreads from $\sim 190 \mathrm{kHz}$ up to $\sim 360 \mathrm{kHz}$. At $70 \mathrm{~km}$ altitude, the first region is located between $\sim 53$ $\mathrm{kHz}$ and $\sim 103 \mathrm{kHz}$, and for $80 \mathrm{~km}$ altitude it starts from $\sim 12 \mathrm{kHz}$ to $\sim 23 \mathrm{kHz}$. Note that these low frequencies depend on the duration of the magnetic field signal therefore they don't have a straightforward physical signification. The second region starts from the previous point to the first bump. At $50 \mathrm{~km}$ altitude, this region reaches up to $\sim 30 \mathrm{MHz}$, for $60 \mathrm{~km}$ altitude it is $9 \mathrm{MHz}$, for $70 \mathrm{~km}$ altitude it is $\sim 3 \mathrm{MHz}$, and for $80 \mathrm{~km}$ altitude it is $\sim 600 \mathrm{kHz}$. The last region is filled with numerical noise. For the cases without collisions, the three regions turn into only two regions. The first region is defined by apower law decrease of the spectral density followed by the second region, which is similar to the third region mentioned above. For $50 \mathrm{~km}$ altitude the steep decrease is in the range 648 $\mathrm{kHz}$ to $30 \mathrm{MHz}$, for $60 \mathrm{~km}$ altitude it is from $193 \mathrm{kHz}$ to $9 \mathrm{MHz}$, for 70 kilometer altitude it begins at $55 \mathrm{kHz}$ and it finishes at $2.6 \mathrm{MHz}$, and for 80 altitude it is between 12 $\mathrm{kHz}$ and $570 \mathrm{kHz}$. For all these altitudes, the spectral density decreases by about a factor of approximately 75000. Note that the frequency associated with the first region is similar to the frequency for the case with collision.

To investigate the capability for radio instruments to detect these electromagnetic signatures, we compare our simulation results to the sensitivity threshold of the instruments IME-HF (dotted black line), FORTE RF payload (dotted dark blue), and ICE (dotted goldenrod) in Figure 4b. In order to make comparison, we use the spectral density but we do not normalize it with respect to a specific antenna length. The sensitivity of IME-HF is slightly dependent on the frequency staying almost constant at $\sim 2 \times 10^{-8}$ $\mathrm{V} / \mathrm{m} / \sqrt{\mathrm{Hz}}$ from $50 \mathrm{kHz}$ to $30 \mathrm{MHz}$, which is significantly below the signal level associated with streamer collisions occurring between $50 \mathrm{~km}$ and $80 \mathrm{~km}$ altitude. On the other hand, the sensitivity for both ICE (30 kHz - $30 \mathrm{MHz})$ and FORTE RF (20-320 MHz) payload are constant in their own frequency range at respectively $10^{-7} \mathrm{~V} / \mathrm{m} / \sqrt{\mathrm{Hz}}$ and $5.6 \times 10^{-8} \mathrm{~V} / \mathrm{m} / \sqrt{\mathrm{Hz}}$. The sensitivity threshold of FORTE is obtained from the upper panel of Plate 1 in Jacobson et al. (1999) indicated to be $\sim 10^{-8.5} \mathrm{~V}^{2} / \mathrm{m}^{2} / \mathrm{MHz}$ in their figure caption. Note that this is an overestimation of the real sensitivity threshold of FORTE (e.g., see Lehtinen et al. (2004), Section 5). The frequency range covered by ICE matches within the frequency range covered by a head-on collision occurring between 50 and 80 
$\mathrm{km}$ altitude. However, its sensitivity threshold is slightly above the two cases at $70 \mathrm{~km}$ and $80 \mathrm{~km}$ altitude. Concerning the FORTE RF payload, its frequency range allows it to observe in the noisy part of the signal where its sensitivity threshold is not sufficient to detect a potential signature of a head-on collision between two streamers. The sensitivity of the two magnetic instruments is not reported because their maximum of sensitivity (which is reached at $1 \mathrm{MHz}$ for IMM and $5 \mathrm{kHz}$ for IMSC) is about 10 times lower than the maximum of the spectral density for a collision-case at $70 \mathrm{~km}$ altitude.

The specific spectral signature depends on the altitude of streamers at the time of the collision (as time scales up with altitude, frequency scales down according to similarity laws (e.g., Pasko, 2006)), thus creating a selective filter. Additionally, note that in this frequency range, the propagation through the ionosphere should reflect a significant part of the VLF-LF signal. We observe in Figure 4b that the sensitivity threshold for IME-HF allows to measure a significant part of the signal associated with a single collision while this proportion is significantly lower for ICE. We hence conclude that for a single head-on collision of double-headed streamers, IMM and IMSC could not detect the signal, while IME-HF and ICE might. Figure 4b shows that the neutral density dependence of streamer collision timescales would make altitude discrimination of events possible, and hence could play a complementary role in the exploitation of photometric measurements. However, note that the cases studied here are under the strong assumption that the effect of the ionosphere is negligible and that the noise context is favorable. If we consider that the nighttime cutoff frequency of the ionosphere is $5 \mathrm{MHz}$ (Davies, 1989), one could conclude that HF signals coming from streamer collisions should not be measurable by the instruments considered in the present study. However, that the ionosphere cutoff frequency depends on the state of the ionosphere, which can be strongly disturbed during thunderstorms, and even ULF-LF radio emissions are known to be observable from space during thunderstorm activity (e.g. Parrot et al., 2008). Moreover, the sensitivity of IME-HF is evaluated from ground based measurements, therefore the true sensitivity in space would only be known in flight.

However, as previously mentioned, sprites are composed of many streamers moving up and down, and often interacting. It is expected that, TARANIS and other groundbased radio instruments will observe an incoherent signal resulting from the complex interactions of many sprite streamers. It is also possible that multiple collisions occurring at the same time would strengthen the radio signal predicted for one single collision in 
the present paper. Furthermore, the radio emission produced by lightning discharges will tend to contaminate data. Extracting relevant information might require to use statistical or machine learning techniques. The observation of the electromagnetic energy released by sprites might help evaluate the number of streamers within sprites, and the importance of their interactions, for example following the study of Liu et al. (2019).

Another parameter having an importance is the angle $\theta$. As previously mentioned, we set it to 90 to maximize the radiated magnetic field (see Equation (6)). We evaluate its influence on the spectral density with respect to sensitivity thresholds of the three instruments. The result is shown in Figure 5.

We note that IME-HF would have been able to detect electromagnetic emissions at $50 \mathrm{~km}$ altitude for $\theta \geq 13^{\circ}$, at $60 \mathrm{~km}$ altitude it becomes $\theta \geq 8^{\circ}$, at $70 \mathrm{~km}$ altitude $\theta \geq 4^{\circ}$, and it drops to $2^{\circ}$ for $80 \mathrm{~km}$ altitude. In the case of ICE due to its sensitivity threshold (see Figure 4), it can only detect a streamer collision at $70 \mathrm{~km}$ altitude with $\theta \geq 29^{\circ}$ and $80 \mathrm{~km}$ altitude for $\theta \geq 13^{\circ}$.It appears that for the case considered here, observations from spacecraft is possible for a broad range of polar angles.

We also evaluate the possibility to observe such events from ground-based telescopes. As an example, the radio telescope NenuFAR performs observations between $10 \mathrm{MHz}$ and $85 \mathrm{MHz}$ (Zarka et al., 2012, 2015). The main scientific objectives regarding NenuFAR are the detection and study of exoplanets in radio, detection of the radio signal of the "Cosmic Dawn" (epoch of formation of the first stars and galaxies), and the study of pulsars (hyperdense and strongly magnetized dead stars in rapid rotation). The typical distance between the radiotelescope and the sprite event is assumed to be similar to that of a satellite observation, such as sketched in Figure 2. In the field of radioastronomy, the Jansky unit ( $\left.1 \mathrm{Jy}=10^{-26} \mathrm{~W} \mathrm{~m}^{-2} \mathrm{~Hz}^{-1}\right)$, corresponding to the electromagnetic spectral flux density, is commonly used to compare the sensitivity of the instrument with sources. Computing the spectral flux density is done through the norm of the Poynting vector $S$ given by:

$$
S=\frac{c B^{2}}{\mu_{0}}
$$

followed by a Fourier transform. Calculations show that the spectral density flux produced by a sprite streamer collision in the NenuFAR range is of $\sim 2$ GJy which is six orders of magnitude larger than the sensitivity of NenuFAR over a timescale of a dozen 
of microseconds, which demonstrates the high potential of radiotelescopes in the study

326 of TLEs.

This article is protected by copyright. All rights reserved. 

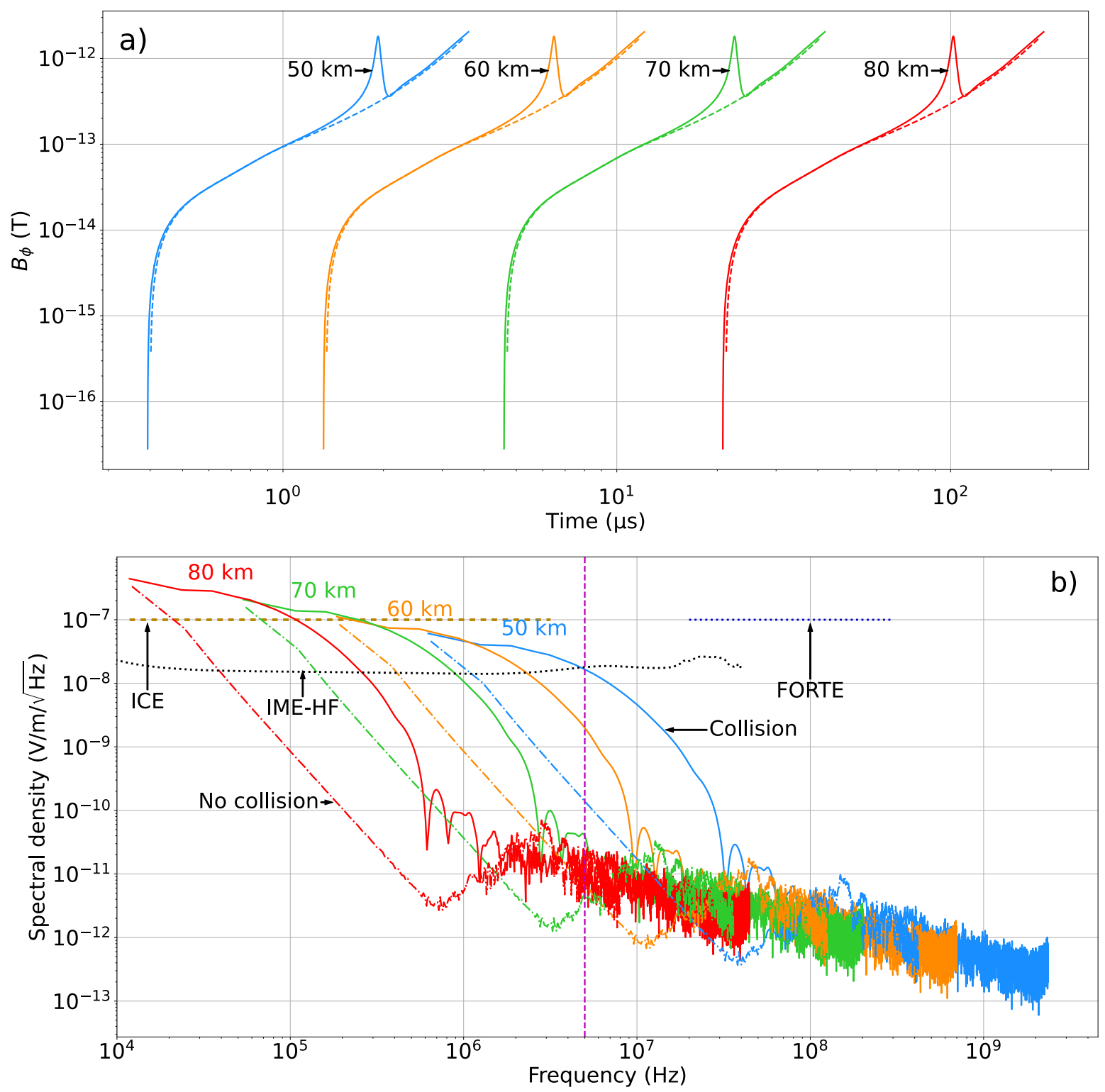

Figure 4. a) Magnetic field radiated by two double-headed streamers evolving at an altitude of $50 \mathrm{~km}$ (blue), $60 \mathrm{~km}$ (orange), $70 \mathrm{~km}$ (green), and $80 \mathrm{~km}$ (red). The peak of each curve appears when the head-on collision between two streamers occurs. b) Spectral density of the electric field radiated by two double-headed streamers experiencing a collision (solid lines) and two, non-interacting streamers (dash-dotted lines) immersed in a homogeneous electric field of 40 $\frac{N}{N_{0}} \mathrm{kV} / \mathrm{cm}$ at $50 \mathrm{~km}, 60 \mathrm{~km}, 70 \mathrm{~km}$, and $80 \mathrm{~km}$ altitude. The dotted black line is for the sensitivity of the electric field instrument (IME-HF) on board TARANIS, while the blue one is for the FORTE RF payload sensitivity and the goldenrod is for the DEMETER radio instrument (ICE). The sensitivity of the magnetic field instrument (IMM) is not shown because its sensitivity is too low (high sensitivity threshold). The dashed vertical magenta line corresponds to the typical ionospheric cutoff nighttime. 


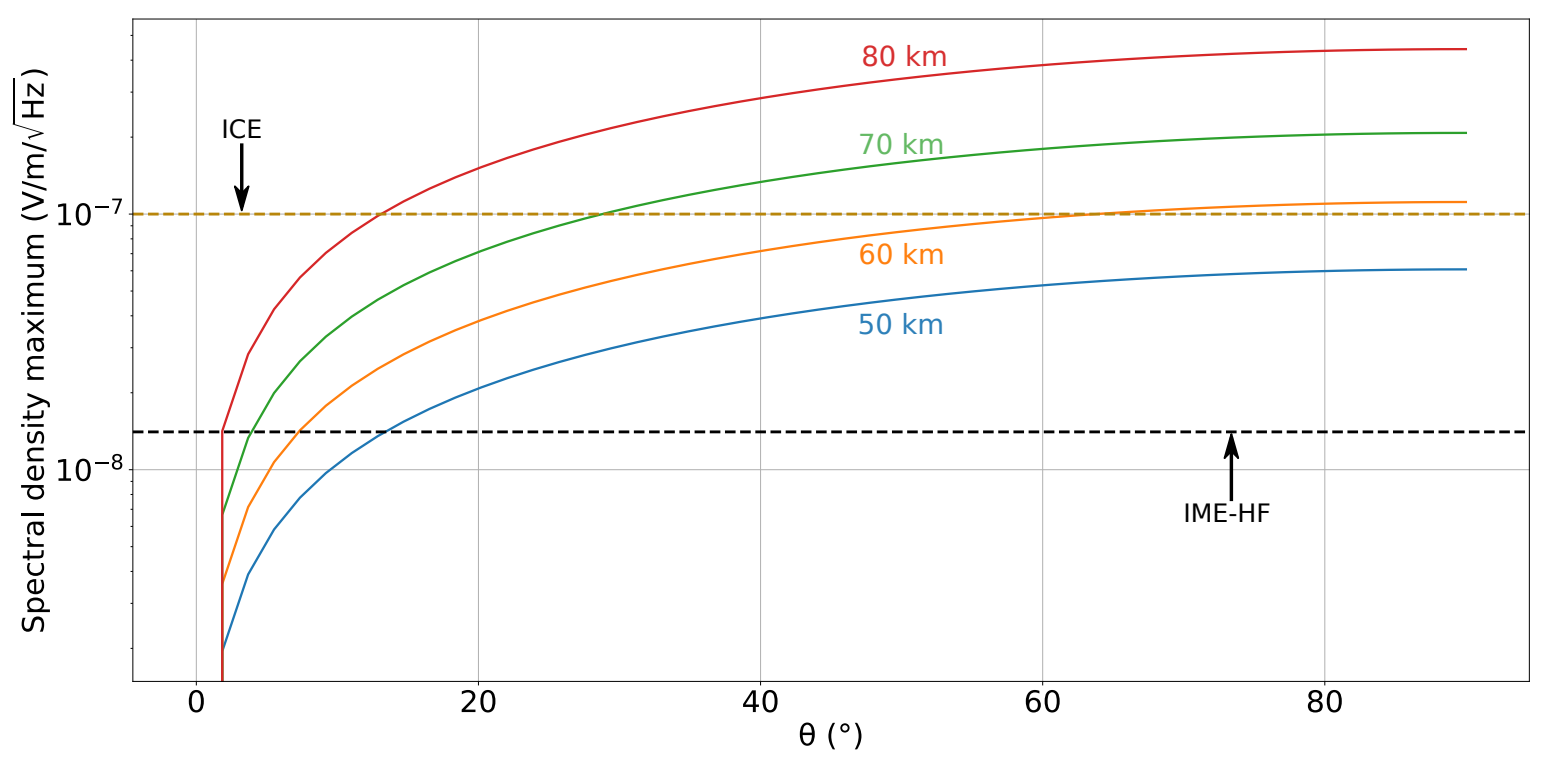

Figure 5. Variation of the maximum of the spectral density as function of the polar angle $\theta$ for a head-on collision at 50 (blue), 60 (orange), 70 (green), and 80 (red) km altitudes. The dotted goldenrod line corresponds to the sensitivity of ICE while the dotted black line is the minimum of the IME-HF sensitivity. 


\section{Conclusions}

In this paper, we investigate the production of electromagnetic radio emissions associated with the interaction of plasma filaments in sprite discharges using a streamer fluid model. We show that the collision between two streamers produces a strong variation of the electric current over a short time scale. The short time scale is caused by the increase of the electron density leading to a high conductivity in the plasma, which then briefly collapses the electric field and produces a strong current variation over this short time scale. We compare the resulting signal with the sensitivity of two radiowave instruments of TARANIS, along with DEMETER and FORTE radio instruments.

For a single collision, we find that the electric field exceeds IME-HF (electric antenna), ICE, and FORTE sensitivity thresholds. However, we conclude that IMM (magnetic antenna) and IMSC sensitivites are too weak to detect such events. The challenge to analyze IME-HF data during thunderstorm where radio emission released by lightning discharges will also be mixing with the radio pattern associated with streamer collisions stays an open question. The results presented in this paper only concern a single head-on collision between two streamers with opposite polarities. This work has a strong implication for the scientific return of a possible revival of the TARANIS mission about the understanding of sprites.

A question remaining open concerns the impact of the ionosphere filtering on the propagation of the signal because in standard condition (i.e., without thunderstorms), the ionosphere tends to block all radio signal below or near its frequency cutoff. However, during a thunderstorm the ionosphere is strongly disturbed and some low-frequency emissions are known to become observable.

In addition to space observations, we find that ground-based instruments like NenuFAR should detect sprite filament collision events, and therefore open a new way to investigate the microphysics of sprites.

Finally, this method is not only applicable to sprite streamers. In principle, the method could be applied to streamers from gigantic jets. In particular, gigantic jets have jump step to the ionosphere at around $50 \mathrm{~km}$ altitude. During that stage, it is possible that descending streamers from the ionosphere collide with ascending streamers produced by the jet (Pasko et al., 2002; da Silva \& Pasko, 2012). 


\section{Acknowledgments}

Authors warmly thank Pr. O. Santolik (IAP) and Dr. J.-L. Rauch (LPC2E) and Dr. J.L. Pinçon (LPC2E) for the discussion concerning the sensitivity of TARANIS radio instruments and the length of the electric antenna. They also thank Dr. L. Bondonneau (LPC2E) and Dr. J.-M. Griessmeier (LPC2E) for the discussion related to the sensitivity of NenuFAR. Authors thank the second reviewer for its suggestion regarding gigantic jets. Data allowing to reproduce the figures of this paper are available from https:// doi.org/10.6084/m9.figshare.13079570.v1

\section{References}

Babich, L., \& Bochkov, E. (2017). Numerical simulation of electric field enhancement at the contact of positive and negative streamers in relation to the problem of runaway electron generation in lightning and in long laboratory sparks. Journal of Physics D: Applied Physics, 50(45), 455202.

Berthelier, J., Godefroy, M., Leblanc, F., Malingre, M., Menvielle, M., Lagoutte, D., ... Pfaff, R. (2006). Ice, the electric field experiment on demeter. Planetary and Space Science, 54(5), 456-471.

Bourdon, A., Pasko, V. P., Liu, N. Y., Célestin, S., Ségur, P., \& Marode, E. (2007). Efficient models for photoionization produced by non-thermal gas discharges in air based on radiative transfer and the Helmholtz equations. Plasma Sources Science and Technology, 16, 656-678.

Cummer, S., Inan, U., Bell, T., \& Barrington-Leigh, C. (1998). ELF radiation produced by electrical currents in sprites. Geophysical Research Letters, 25, 12811284 .

Cummer, S. A., Frey, H. U., Mende, S. B., Hsu, R., Su, H., Chen, A. B., .. Takahashi, Y. (2006). Simultaneous radio and satellite optical measurements of high-altitude sprite current and lightning continuing current. Journal of Geophysical Research: Space Physics, 111, A101315.

da Silva, C. L., \& Pasko, V. P. (2012). Simulation of leader speeds at gigantic jet altitudes. Geophysical Research Letters, 39(13), L13805.

Davies, K. (1989). Ionospheric radio. P. Peregrinus, London.

Farges, T., \& Blanc, E. (2011). Lightning and tle electric fields and their impact on the ionosphere. Comptes Rendus Physique, 12(2), 171 - 179. 
Farges, T., Hébert, P., Le Mer-Dachard, F., Ravel, K., \& Gaillac, S. (2018). Microcameras and photometers (MCP) on board the TARANIS satellite. In XVI International Conference on Atmospheric Electricity.

Füllekrug, M., Moudry, D. R., Dawes, G., \& Sentman, D. D. (2001). Mesospheric sprite current triangulation. Journal of Geophysical Research: Atmospheres, 106 (D17), 20,189-20,194.

Füllekrug, M., Roussel-Dupré, R., Symbalisty, E. M. D., Chanrion, O., Odzimek, A., van der Velde, O., \& Neubert, T. (2010). Relativistic runaway breakdown in low-frequency radio. Journal of Geophysical Research: Space Physics, 115, A00E09.

Franz, R., J Nemzek, R., \& R Winckler, J. (1990). Television image of a large upward electrical discharge above a thunderstorm system. Science, 249, 48-51.

Gerken, E. A., Inan, U. S., \& Barrington-Leigh, C. P. (2000). Telescopic imaging of sprites. Geophysical Research Letters, 27, 2637-2640.

Golub, G. H., \& Van Loan, C. F. (1996). Matrix computations (4rd ed.). Baltimore, Maryland, USA: Johns Hopkins University Press.

Ihaddadene, K. M. A., Dwyer, J. R., Liu, N., Celestin, S., \& Shi, F. (2019). Modeling of a new electron acceleration mechanism ahead of streamers. Journal of Geophysical Research: Space Physics, 124(8), 7301-7319.

Ihaddadene, M. A., \& Celestin, S. (2015). Increase of the electric field in head-on collisions between negative and positive streamers. Geophysical Research Letters, 42, 5644-5651.

Jacobson, A. R., Knox, S. O., Franz, R., \& Enemark, D. C. (1999). Forte observations of lightning radio-frequency signatures: Capabilities and basic results. Radio Science, 34(2), 337-354.

Köhn, C., Chanrion, O., \& Neubert, T. (2017). Electron acceleration during streamer collisions in air. Geophysical Research Letters, 44(5), 2604-2613.

Lefeuvre, F., Blanc, E., Pinçon, J.-L., Roussel-Dupré, R., Lawrence, D., Sauvaud, J.-A., ... Lagoutte, D. (2008). Taranis - a satellite project dedicated to the physics of tles and tgfs. In Planetary atmospheric electricity (pp. 301-315). Springer New York.

Lehtinen, N. G., Gorham, P. W., Jacobson, A. R., \& Roussel-Dupré, R. A. (2004). Forte satellite constraints on ultrahigh energy cosmic particle fluxes. Physical 
Review D, 69(1), 013008.

Liu, N., Dwyer, J. R., Tilles, J. N., Stanley, M. A., Krehbiel, P. R., Rison, W., ... Wilson, J. G. (2019). Understanding the radio spectrum of thunderstorm narrow bipolar events. Journal of Geophysical Research: Atmospheres, 124(17-18), 10134-10153.

Liu, N., \& Pasko, V. P. (2004). Effects of photoionization on propagation and branching of positive and negative streamers in sprites. Journal Geophysical Research, 109, A04301.

Liu, N. Y., Pasko, V. P., Adams, K., Stenbaek-Nielsen, H. C., \& McHarg, M. G. (2009). Comparison of acceleration, expansion, and brightness of sprite streamers obtained from modeling and high-speed video observations. Journal of Geophysical Research: Space Physics, 114, A00E03.

Luque, A. (2017). Radio frequency electromagnetic radiation from streamer collisions. Journal of Geophysical Research: Atmospheres, 122(19), 10,497-10,509.

Luque, A., \& Ebert, U. (2010). Sprites in varying air density: Charge conservation, glowing negative trails and changing velocity. Geophysical Research Letters, 37(6), L06806.

Luque, A., \& Gordillo-Vázquez, F. J. (2011). Sprite beads originating from inhomogeneities in the mesospheric electron density. Geophysical Research Letters, 38(4), L04808.

McHarg, M. G., Maldonado, C., Taylor, L. M., da Silva, C. L., Vidal, L. C., Stenbaek-Nielsen, H., ... Haaland, R. K. ～(2019). Sprite streamer interactions at 100,000 frames per second, AE23A-08. In AGU Fall Meeting, 9-13 december 2019.

McHarg, M. G., Stenbaek-Nielsen, H. C., Kanmae, T., \& Haaland, R. K. (2010). Streamer tip splitting in sprites. Journal of Geophysical Research: Space Physics, 115, A00E53.

Morrow, R., \& Lowke, J. J. (1997). Streamer propagation in air. Journal of Physics D: Applied Physics, 30, 614.

Parrot, M., Benoist, D., Berthelier, J., Błęcki, J., Chapuis, Y., Colin, F., ... Zamora, P. (2006). The magnetic field experiment imsc and its data processing onboard demeter: Scientific objectives, description and first results. Planetary and Space Science, 54(5), 441-455. 
Parrot, M., Inan, U. S., \& Lehtinen, N. G. (2008). V-shaped VLF streaks recorded on DEMETER above powerful thunderstorms. Journal of Geophysical Research: Space Physics, 113, A10310.

Pasko, V. P. (2006). Theoretical modeling of sprites and jets, in sprites, elves and intense lightning discharges. NATO Sci. Ser., Springer, Heidleberg, Germany.

Pasko, V. P., Inan, U. S., \& Bell, T. F. (1998). Spatial structure of sprites. Geophysical Research Letters, 25, 2123-2126.

Pasko, V. P., Stanley, M. A., Mathews, J. D., Inan, U. S., \& Wood, T. G. $\quad$ (2002). Electrical discharge from a thundercloud top to the lower ionosphere. Nature, $416,152-154$.

Press, W. H., Teukolsky, S. A., Vetterling, W. T., \& Flannery, B. P. (2007). Numerical recipes 3rd edition: The art of scientific computing (3rd ed.). New York, NY, USA: Cambridge University Press.

Qin, J., Celestin, S., \& Pasko, V. P. (2012). Low frequency electromagnetic radiation from sprite streamers. Geophysical Research Letters, 39, L22803.

Qin, J., \& Pasko, V. P. (2015). Dynamics of sprite streamers in varying air density. Geophysical Research Letters, 42(6), 2031-2036.

Shi, F., Liu, N., Dwyer, J. R., \& Ihaddadene, K. M. A. (2019). VHF and UHF electromagnetic radiation produced by streamers in lightning. Geophysical Research Letters, 46(1), 443-451.

Uman, M., Mclain, K., \& Krider, E. (1975). The electromagnetic radiation from a finite antenna. American Journal of Physics, 43, 33-38.

Vetter, S. (2019). https://apod.nasa.gov/apod/ap191008.html. (Consultation on 2nd Sep. 2020, 14h37)

Zalesak, S. T. (1979). Fully multidimensional flux-corrected transport algorithms for fluids. Journal of Computational Physics, 31(3), 335 - 362.

Zarka, P., Girard, J. N., Tagger, M., Denis, L., \& the LSS team. LSS/NENUFAR: The LOFAR super station project in Nançay. In SF2A-2012: Semaine de l'astrophysique francaise (pp. 687-694).

Zarka, P., Tagger, M., Denis, L., Girard, J. N., Konovalenko, A., Atemkeng, M., ... Zakharenko, V. (2015). NenuFAR: Instrument description and science case. In International conference on antenna theory and technique (pp. 13-18). 